Revue d'histoire de l'enfance « irrégulière »

Le Temps de l'histoire

$3 \mid 2000$

L'enfant de justice pendant la guerre et l'immédiat après-guerre

\title{
La loi du 27 juillet 1942 ou l'issue d'une querelle de monopole pour l'enfance délinquante
}

\author{
(2) OpenEdition \\ Journals \\ Édition électronique \\ URL : http://journals.openedition.org/rhei/71 \\ DOI : $10.4000 /$ rhei. 71 \\ ISBN : 978-2-7535-1640-3 \\ ISSN : $1777-540 \mathrm{X}$ \\ Éditeur \\ Presses universitaires de Rennes \\ Édition imprimée \\ Date de publication : 15 novembre 2000 \\ Pagination : $55-76$ \\ ISSN : 1287-2431

\section{Référence électronique} \\ Michèle Becquemin-Girault, «La loi du 27 juillet 1942 ou l'issue d'une querelle de monopole pour \\ l'enfance délinquante », Revue d'histoire de l'enfance « irrégulière » [En ligne], $3 \mid$ 2000, mis en ligne le 30 \\ avril 2007, consulté le 04 décembre 2020. URL : http://journals.openedition.org/rhei/71 ; DOI : https:// \\ doi.org/10.4000/rhei.71
}




\section{La loi du 27 juillet 1942}

\section{ou l'issue d'une querelle de monopole pour l'enfance délinquante}

Depuis plus d'un siècle, et parallèlement à l'œuvre scolaire, des lois successives ont organisé le dispositif de protection de l'enfance dans un double mouvement : enrayer la morbidité infantile et résorber la criminalité juvénile. Depuis lors, de nombreuses institutions se partagent le domaine de l'enfance à protéger en fonction de leurs missions et de leurs spécificités.

Sur le versant du droit pénal des mineurs, les législations de 1912, 1935 et 1945 sont considérées comme des étapes importantes, en particulier dans les affiliations faites par le législateur qui, à l'aube de la Vème République, remania les structures du dispositif ${ }^{(2)}$ dans le but de constituer un "système complet de protection de l'enfance". Mais, alors qu'il se référait aux dispositions financières et institutionnelles de la loi du 3 juillet 1944, promulguée avant la fin du régime de Vichy, il ne faisait, en revanche, aucun cas de la loi du 27 juillet $1942,{ }^{(3)}$ destinée à créer des tribunaux pour enfants et adolescents dotés de "centres d'observation". ${ }^{(4)}$ Cette loi annonçait pourtant, avec quelques singularités conjoncturelles, des orientations juridiques et organisationnelles qui s'établirent dans les années qui suivirent pour constituer les charpentes du dispositif actuel. ${ }^{(5)}$ Malgré ses intentions réformistes, la loi de 1942 fut en effet l'objet d'une relative amnésie. Il en reste peu de traces dans les pratiques comme dans les écrits. Abrogée à la Libération, elle fut, sur certains points, refondue dans l'ordonnance de 1945. Son impact institutionnel fut faible pendant les trois années de sa mise en vigueur, hormis des dispositions hâtives au sein de l'Éducation surveillée, ${ }^{(6)}$ dont certaines ont d'ailleurs précédé sa promulgation.

Comprendre l'élaboration de cette législation "évanouie" oblige le
Michèle BecqueminGirault $^{(1)}$

(1) Éducatrice au

Service social de

l'enfance de Paris, doctorante en sociologie.

(2) Ministère de la Justice, La protection de l'enfance en danger, Vaucresson, mai 1960, pp. 9-14.

(3) Loi n683 du 27 juillet 1942, JO, 13 août 1942 , p. 2778 , rectifiée le 25 août 1942.

(4) Ibid., Art. 27 :

Centres d'observation.

(5) Michel CHAU-

VIÈRE, Enfance

inadaptée : l'béritage de

Vichy, Les éditions

ouvrières, Paris, 1987.

(6) Christian SAN-

CHEZ, "Les centres d'accueil et de triage de l’Éducation surveillée 1941-1950”, le Temps de l'histoire, ${ }^{\circ} 1$, février

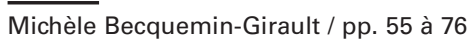


1998, CNFE-PJJ

Vaucresson, p. 121.

(7) Je remercie Michel Chauvière et Jacques Bourquin pour leurs conseils et leurs indications, ainsi que Patricia Gillet, conservateur des Archives nationales, pour ses orientations.

(8) Selon l'avis de d'Hélène Campinchi, avocate à la Cour d'appel de Paris, juriste auprès de patronages et de services sociaux de la Seine.

(9) Propos d'Henry BERTHELEMY, doyen de la faculté de Droit à Paris, cité par Jean BANCAL, Essai sur le redressement de l'enfance coupable, Paris, Sirey, 1941, p. 38.

(10) En 1906, la minorité pénale était passée de 16 à 18 ans. Cette loi stipulait que l'excuse de minorité ne s'appliquait pas aux 16-18 ans. Elle "préfabriquait" ainsi la catégorie des 16-18 ans qui subsista en 1912 . chercheur à se confronter au problème des sources ${ }^{(7)}$ disparues ou éparpillées émanant du gouvernement de Vichy, qui légiférait à partir de décisions prises par des hauts techniciens sous l'égide du maréchal Pétain. Une explication exhaustive nécessiterait des recherches approfondies ; je n'en proposerai ici qu'un éclairage partiel. Il délaissera les questions concernant l'application institutionnelle de la loi (création de tribunaux pour enfants et de centres d'observation), pour mieux montrer comment les caractéristiques de la législation furent conditionnées par les enjeux politiques et institutionnels qui présidèrent à son avènement.

\section{Les objectifs de la réforme}

La loi du 27 juillet 1942 fut confectionnée pour remplacer la législation de 1912, estimée, pour le moins, désuète sur certains points ${ }^{(8)}$ et qualifiée, pour le pire, de duperie. ${ }^{(9)}$ La nouvelle législation procédait à quelques innovations remarquables. Elle accentuait l'autonomie du droit pénal de l'enfance et affirmait la spécialisation des juridictions des mineurs qui existaient seulement dans quelques grandes villes, tout en préconisant des réformes quant aux méthodes de redressement et aux établissements de l'Éducation surveillée. Un regard rétrospectif la jugerait complexe. En fait, l'objectif de cette loi consistait à simplifier le système de 1912, trop compliqué, trop lent, trop subtil. Pour ce faire, la loi de 1942 cherchait autant à unifier certains principes qu'à établir des arrangements pragmatiques en intégrant les difficultés du moment : inflation de la délinquance, pénurie de moyens institutionnels et financiers.

En premier lieu, le législateur avait le souci d'uniformiser le régime des mineurs. En effet, la loi de 1912 avait institué de nouvelles catégories : les moins de 13 ans, les 13-16 ans et les 16-18 ans. ${ }^{(10)}$ Les mineurs de 13 ans devaient obligatoirement faire l'objet d'une information préalable au jugement et la question du discernement pour cette tranche d'âge avait été supprimée, dès 1912, au profit d'une présomption légale et irréfragable d'irresponsabilité pénale. En 1942, la nouvelle législation cherchait à établir des principes communs à tous les mineurs de 18 ans, sur les bases de 1912 qu'elle modifiait partiellement. Ainsi, elle instaurait une procédure qui exigeait une information préalable avant d'enga- 
ger des poursuites quel que soit l'âge de l'enfant. ${ }^{(1)}$ En outre, elle sup(11) Loi n 683 du 27 primait complètement la notion du discernement restée en vigueur pour les mineurs de 13 à 18 ans, en raison d'un usage jugé "prétorien" de ce critère. Parallèlement, elle faisait disparaître la présomption irréfragable d'irresponsabilité pénale portant sur les moins de 13 ans, pour établir en général la primauté des mesures de protection et de redressement. ${ }^{(12)}$ Tous les mineurs de moins de 18 ans bénéficiaient donc, en principe, du même régime, mais la loi de 1942 conservait, en exception à ce principe, les délinquants et les criminels âgés de 16 à 18 ans, pour lesquels elle préconisait des sanctions graduellement répressives, en les assimilant pénalement aux majeurs. ${ }^{(13)}$ Cette mesure avait été maintenue dans le souci de sauvegarder l'ordre public, préoccupation dans laquelle s'inscrivaient aussi les dispositions prévues à l'encontre des adultes coauteurs des crimes ou des délits commis par les mineurs.

Un deuxième objectif, également lié à l'accroissement du nombre de délits, s'était imposé : il fallait filtrer les flux d'enfants susceptibles d'être jugés. Pour hâter le traitement des affaires, autant que pour rendre plus performantes les tâches des juridictions spécialisées, la procédure avait été conçue en trois temps. ${ }^{(14)}$ Le premier correspondait à la phase de l'instruction au terme de laquelle le mineur devait, si nécessaire, comparaître devant une juridiction civile de première instance, la chambre du conseil. Ensuite, cette juridiction devait étudier le cas de l'enfant et statuer sur l'opportunité d'une mise en accusation. Le cas échéant, la chambre du conseil saisissait le tribunal pour enfants et adolescents. Le tribunal ne devait intervenir que dans le troisième temps de la procédure. Ainsi, l'idée de 1912, qui consistait à répartir les jugements en distinguant les cas par tranches d'âge (moins de 13, 13-16 et 16-18 ans) et par le degré de responsabilité, disparaissait dans une réorganisation des juridictions pour une meilleure distribution des flux d'enfants.

Dans la lignée des réformes juridiques antérieures, la loi prônait d'individualiser les décisions. Pour y parvenir, deux moyens avaient été retenus : la spécialisation de la juridiction ${ }^{(15)}$ et l'observation approfondie du mineur. ${ }^{(16)}$ La loi ordonnait en effet la création de tribunaux spécialisés, dont la répartition géographique devait être fixée ultérieurement, par

juillet 1942, JO, 13 août 1942, p. 2778, Art. 3.

(12) Ibid., Art. 17.

(13) Ibid., Art. 23.

(14) Ibid., Art. 2, 7 et 10 .

(15) Ibid., Art. 14.

(16) Ibid., Art. 8 et 27. 
(17) Dr Gilbert

ROBIN, “L'enfant nerveux, retardé et instable, Revendications législatives - Réformes indispensables”, Le siècle médical, 1er mai 1929.

(18) JO, 13 août 1942, "Rapport au maréchal de France, chef de l'État français", (préambule à la) Loi n $683 \mathrm{du} 27$ juillet 1942 .

(19) Ibid., Art. 22.

(20) Ibid. décret. Le tribunal devait être composé d'un magistrat de cour d'appel et de deux magistrats de première instance. Ceux-ci étaient assistés de deux assesseurs, hommes ou femmes, en cas de crime seulement.

Les tribunaux devaient fonctionner en lien étroit avec des centres d'observation situés à proximité, dont la loi prévoyait également la création. L'observation devait être suivie de près par un magistrat, jusqu'aux résultats qui permettraient de statuer. Déjà présente dans l'esprit du législateur de 1912, l'idée d'observer le mineur pour une meilleure adéquation du jugement n'avait été concrétisée que par les enquêtes et les examens médicaux pour les mineurs de 13 ans. Le vœu de créer des centres d'observation avait ensuite été formulé en 1925 par la 4ème sous-commission de réforme pénitentiaire, à la suite d'un rapport effectué par le Dr Paul-Boncour. ${ }^{(17)}$ Parallèlement, certains règlements avaient amené l'idée de "triage" (circulaire de 1926, décret de 1929, circulaire de 1935). Le décret-loi de 1935 prévoyait un placement préventif pendant la durée de l'enquête sociale et de l'examen médical pour les mineurs vagabonds. Dans les pays voisins, des centres d'observation avaient été institués dans la foulée des innovations concernant les juridictions des mineurs. C'est le modèle italien qui inspira le législateur de 1942. ${ }^{(18)}$

La nouvelle loi reprenait en outre la disposition de 1912 selon laquelle les jugements pouvaient être revus en fonction de l'évolution de la situation du mineur. ${ }^{(19)}$

L'Éducation surveillée devait servir de modèle. ${ }^{(2)}$ La loi prévoyait des réformes au niveau de ses structures, de son personnel et des méthodes de redressement. Elle préconisait la création d'établissements qui serviraient de guides aux œuvres privées. Les appuis cognitifs proviendraient des sciences médicales, de la pédagogie et de l'expérience acquise dans les mouvements de jeunesse.

Enfin, la législation permettait de recourir à une grande variété de ressources institutionnelles, tout en préconisant leur coordination. A chacune des étapes de l'instruction et du jugement, il était possible d'utiliser des mesures diversifiées et une large gamme de services et d'œuvres appartenant aux secteurs public et privé. Le choix des établissements était laissé à l'appréciation des juges. Plutôt qu'un classement par caté- 
gories de mineurs établi sur des compétences institutionnelles, l'organisation prônée privilégiait une distribution souple mais coordonnée des flux d'enfants, incluant des possibilités de réciprocité entre l'Éducation surveillée et les autres institutions. Les établissements étaient soumis à des contrôles ${ }^{(21)}$ administratifs ; les œuvres privées devaient en outre obtenir l'habilitation du ministère de la Justice. ${ }^{(22)}$

Les activités de ce dispositif se déroulaient sous l'égide d'une coordination interministérielle placée sous l'autorité du Chef de l'État. Les signataires de cette loi - Joseph Barthélemy (Justice), Pierre Cathala (Finances), Raymond Grasset (Santé), Abel Bonnard (Éducation nationale), l'Amiral Platon (Famille) - représentaient les pôles de cette coordination.

\section{Les points sensibles}

Sous le peigne fin des juristes de l'époque, ${ }^{(23)}$ les points sensibles du texte législatif devenaient de véritables paradoxes. Ils apparaissaient soit en raison d'un manque de précision par rapport aux dispositions antérieures abrogées par la législation (mais non remplacées), soit dans des contradictions entre l'intention législative et son projet d'application.

Par exemple, l'élimination de l'irresponsabilité totale en faveur des moins de 13 ans laissait supposer qu'un enfant de 6, 7 ou 8 ans pouvait, dans certains cas et en dépit d'une excuse atténuante de minorité, encourir une condamnation qui n'excluait pas la peine de mort.

Quant à la suppression de la notion de discernement, elle équivalait à faire disparaître les possibilités d'acquittement auparavant attribuées en cas de non discernement. Parallèlement, le traitement infligé aux 1618 ans par l'article 23 était singulièrement répressif dans l'esprit. Cependant, la loi omettait de préciser les applications des peines encourues (peine de mort, condamnation à perpétuité, déportation), pourtant suggérées par l'assimilation de cette tranche d'âge aux majeurs. Comparativement aux dispositions antérieures de l'article 67 du code pénal dorénavant abrogé, les articles 23 et 39 de la nouvelle loi n'apportaient aucune précision sur ce chapitre. Ces châtiments et leurs lacunes juridiques sont à mettre en lien avec les hésitations qui subsistaient depuis
(21) Mesures datant de 1933 et 1938 en ce qui concerne les œuvres privées.

(22) JO, 13 août 1942, Loi $\mathrm{n}^{\circ} 683$ du 27 juillet 1942, Art. 31.

(23) Voir les articles de DONNEDIEU DE

VABRES, Hélène

CAMPINCHI et Michel BOITARD dans la revue Etudes de science criminelle et droit comparé, Sirey, Paris, 1945. 
(24) Hélène CAMPINCHI, "La majorité pénale”, Union des sociétés de patronage, Recueil des bulletins 1941 1943.
1906, lorsque la majorité pénale était passée de 16 à 18 ans. ${ }^{(24)}$ Dès qu'il y avait une nouvelle inflation de criminalité, les questions du traitement des 16-18 ans et de l'abaissement de la majorité pénale étaient de nouveau agitées. Les lois de janvier et d'avril 1941 sur "les stages obligatoires dans les chantiers de jeunesse" et les mesures prises à l'encontre des plus de 16 ans auteurs d'agressions nocturnes avaient d'ailleurs été conçues dans cet ordre d'idées.

Si la loi avait pour vocation de favoriser l'investigation sur le milieu et la personnalité du mineur et d'individualiser son traitement, elle ne se donnait pas les garanties de son application. D’une part, les enquêtes sociales et l'examen médical ne revêtaient aucun caractère obligatoire. D'autre part, l'intervention de la juridiction spécialisée et l'utilisation des centres d'observation n'entraient en jeu qu'en dernière instance (de même que le recours à la liberté surveillée), ce qui alourdissait la procédure pour les mineurs concernés.

Le texte ne supprimait pas complètement le recours aux maisons d'arrêt pendant l'instruction, reprenant ainsi l'ambiguïté de la circulaire du 21 mars 1942. Il réduisait considérablement les possibilités de défense et d'appel, en comparaison des dispositions de 1912. Enfin, une grande prudence était observée quant au nombre et à la répartition des structures que la loi se proposait de créer, alors qu'elle faisait preuve d'une large possibilité de connexions avec des institutions non judiciaires.

Comment expliquer ces paradoxes par rapport aux intentions ostensiblement réformistes de cette loi ? Dans quelles mesures ces paradoxes étaient-ils liés au contexte social, économique et politique du régime de Vichy ? Ont-ils été les aboutissements fâcheux de divergences de points de vue qui auraient opposé les auteurs du texte législatif ?

Une investigation plus poussée révèle en effet que deux groupes d'acteurs étaient alors concernés par le problème de l'enfance délinquante.

\section{Les prétendants à l'enfance délinquante}

Le texte législatif de 1942 peut être considéré comme l'aboutissement de nombreux travaux antérieurs qui visaient à réformer la prise en charge de l'enfance appelée jusque-là “coupable”. Des projets et des propo- 
sitions de loi apparurent à foison : Rollin (1935), Lagarde (1936), Rucart (1937), Delattre (1937), Campinchi (1937), Matter (1938).

Mais cet élan réformiste ne fut pas constitué d'un rassemblement de volontés unanimes. Plusieurs courants issus de rivalités institutionnelles superposées et cristallisées au début du siècle ${ }^{(25)}$ restaient en opposition et s'adressaient mutuellement de sévères critiques de légitimité dont on continue à mesurer l'héritage aujourd'hui. En résumé, la question du traitement de l'enfance coupable réactivait les rivalités entre un corps judiciaire devenu influent dès le début de la Troisième République et une Administration pénitentiaire exsangue quant aux moyens dont elle disposait. Au premier groupe était allié une grande partie du secteur privé associatif ${ }^{\left({ }^{26)}\right.}$ concerné par l'enfance malheureuse, déficiente ou coupable, sur lequel se greffaient les prérogatives naissantes du ministère de la Santé, qui assumait l'héritage de l'Assistance publique, tout en s'équipant d'experts médicaux dans le domaine de la psychiatrie infanto-juvénile. Les lois de 1889 et de 1898 avaient entériné des changements de conceptions : dorénavant les enfants coupables étaient en premier lieu des victimes d'un milieu familial indigne ou carencé. Pour les milieux sociojudiciaires, l'enjeu principal était d'intégrer l'enfance coupable dans le lot des enfants en danger moral, soignables, rééducables. Pour les fonctionnaires de l'Administration pénitentiaire, il s'agissait de garder cette population en la maintenant au statut de catégorie spécifique "d'enfance délinquante", au prix de certaines réformes institutionnelles.

\section{- Le courant socio-judiciaire}

Le secteur associatif était issu des mouvements philanthropiques (Société générale des prisons, Sociétés de patronage, Comités de défense des enfants traduits en Justice). L'orientation des activités était préventive $^{(27)}$ : éviter la récidive après détention ou éviter l'emprisonnement. Les juges du tribunal de la Seine, tels Henri Rollet, l'un des promoteurs de la loi de 1912, puis le substitut Baffos, avaient été actifs dans la dépénalisation du vagabondage, la transformation de la correction paternelle et de la déchéance de puissance paternelle en assistance éducative en 1935. Les pratiques mises en œuvre par les services sociaux
(25) Voir Henri

GAILLAC, Les maisons

de correction, 1830-1945,

éd. Cujas, Paris, 1991, pp.157-159, pour plus de clarté sur cette question complexe qui intègre les politiques anti-cléricales des républicains, les variations dans les redistributions des charges du ministère de l'Intérieur à la fin du XIXème, le rattachement de l'Administration pénitentiaire au ministère de la Justice en 1911, la naissance du ministère de la Santé en 1920, et leurs impacts sur la répartition des effectifs d'enfants dans les institutions.

(26) Avant la guerre, les magistrats confiaient quatre fois plus de mineurs aux œuvres privées qu'à l'Administration pénitentiaire.

(27) Michèle BECQUEMIN-GIRAULT, Protection de l'enfance et dynamique préventive, Mémoire de DEA de sociologie, sous la direc- 
tion de Michel Chauvière, EHESS, 1998.

(28) Voir Michel CHAUVIÈRE, op. cit., pp. 35-37 et 47-49. attachés aux tribunaux avaient progressivement infléchi les conceptions en matière de délits commis par les mineurs. L'idée que ces enfants étaient avant tout "en danger moral" et qu'il fallait les "préserver" s'imposait progressivement. Les méthodes de redressement des mineurs étaient inspirées des techniques du case-work. Les cas passaient par un examen du milieu familial, éventuellement une consultation médicopsychologique et, au besoin, un placement en foyer d'observation et de rééducation. Ces pratiques existaient principalement dans le département de la Seine où s'activaient quatre services sociaux spécialisés. Parmi eux, le Service social de l'enfance avait ouvert le foyer de Soulins, dès 1929, pour l'observation et la rééducation des enfants. D'autres initiatives avaient pris forme dans les grandes villes de province, souvent dans la continuité d'un patronage. Ces interventions visaient le relèvement des parents défaillants, une scolarité régulière, des prises en charge adaptées aux anormaux et un reclassement par le travail. Elles se conjuguaient avec des œuvres de rééducation, généralement d'origine confessionnelle, dont les méthodes variaient selon les ressources locales et les convictions des fondateurs, par exemple : l'ESSOR occitan, fondé par l'abbé Plaquevent en région toulousaine, le Sauvetage de l'enfance de Lyon, les services et établissements du Hinglé en Bretagne, dirigés par Mme de la Morlais. ${ }^{(28)} \mathrm{Ce}$ courant recevait l'appui technique et scientifique des personnalités de la neuropsychiatrie infantile, dont un des principaux représentants était le Dr Georges Heuyer, à Paris. Des experts en droit, Pierre de Casabianca, Donnedieu de Vabres, pour ne citer qu'eux, offraient à ce mouvement le profit de leurs influences. Politiquement, toutes ces initiatives s'inscrivaient globalement dans les idées de la démocratie chrétienne. En outre, une œuvre multiconfessionnelle comme le Service social de l'enfance en danger moral de Paris était également soutenue par les milieux socialistes et par des juristes comme Mme Campinchi, fille du député radical socialiste corse, Adolph Landry, et épouse de César Campinchi, auteur d'un projet de loi en 1937.

\section{- Le courant réformiste de l'Éducation surveillée}

D'un autre côté, les fonctionnaires de la Pénitentiaire, dont l'inspec- 
teur général Jean Bancal, préconisaient d'émanciper l'Éducation surveillée de l'Administration pénitentiaire, pour en réformer les méthodes et l'organisation des établissements. Ces mesures s'imposaient car, au lendemain de la première guerre, l'équipement de l'Administration pénitentiaire était détérioré et unanimement critiqué. En répercussion, sur les quinze structures d'avant-guerre, six avaient été fermées. Une baisse considérable des effectifs d'enfants ${ }^{(29)}$ avait eu lieu au profit des patronages. Par ailleurs, suite au succès des romans d'Édouard Quet ${ }^{(30)}$ et d'André Lorde ${ }^{(31)}$ qui avaient précédé la législation de 1912, la presse et l'opinion étaient restées sensibles au sort des enfants difficiles ou malheureux. Pendant les années 1920, des journalistes avaient entrepris de grandes enquêtes sur les traitements de l'enfance malheureuse, coupable et déficiente. En 1924, Louis Roubaux avait fait paraittre des reportages dans le Quotidien de Paris, après avoir visité Eysses, Aniane, Belle-Ile, Clermont et Doullens. Il avait déclaré que "ces écoles professionnelles [étaient] tout simplement des écoles du bagne $"{ }^{(32)}$ Trois ans plus tard, Raymond de Nys avait consacré à "l'enfance maudite » ${ }^{(3)}$ une série d'articles dans le journal Le petit parisien. Ce journaliste était favorable aux patronages. Concernant l'Éducation surveillée, il considérait " qu'il n'y avait plus guère de tortionnaires dans ces maisons d'enfants mais qu'il fallait surtout éduquer le personnel de surveillance $»{ }^{(34)}$ En 1929, Alexis Danan, alors journaliste à Paris soir, réalisa également une enquête dans laquelle il rendait compte, en 17 articles, des différentes initiatives de prise en charge institutionnelle des «enfants anormaux ${ }^{(3)}$ en France, en Suisse et en Autriche. Lors de son enquête, M. Mouton, directeur des Affaires criminelles à la Justice, M. Cazeaux à l'Intérieur et M. Estèves à la Pénitentiaire lui avaient refusé l'autorisation de visiter une maison de correction. Piqué, Alexis Danan se rendit néanmoins, grâce à la recommandation d'un juge, dans un établissement de correction. Ce reportage quasi clandestin lui inspira son dixième article, intitulé "La maison des méchants garçons". ${ }^{(3)}$ Il en dénonçait l'aspect disciplinaire et y voyait un lieu de "perdition définitive ». Par la suite, il fut l'instigateur virulent des campagnes de presse contre les bagnes d'enfants en 1934, puis en avril $1937^{(37)}$ à la suite du décès d'un colon à la colonie d'Eysses.
(29) Henri GAILLAC, op. cit., pp. 261-264.

(30) Edouard QUET,

En correction, Bibliothèque Charpentier, E. Fasquelle, 1906, in $8^{\circ}, 310 \mathrm{p}$.

(31) André LORDE, Pierre CHAINE, Bagnes d'enfants. Drame en quatre actes... d'après le roman :

En correction de

M. Edouard QUET, imprimerie de l'illustration théâtrale, Paris, 1910, in $4^{\circ}, 36 \mathrm{p}$.

(32) Henri GAILLAC, op. cit., p. 280.

(33) Raymond DE NYS, "L'enfance maudite", Le petit parisien, du 22 décembre $1927 \mathrm{au}$ 23 janvier 1928.

(34) Raymond DE NYS, op. cit., 31 décembre 1927.

(35) Alexis DANAN, "La grande pitié des enfants anormaux", Paris Soir, du 19 février 1929 au 10 mars 1929.

(36) Alexis DANAN, op. cit., 28 février 1929.

(37) AN, BB 301703. 
(38) Pierre PEDRON, La prison sous Vichy, éd. de l'Atelier, Paris, 1996, p. 88 .

(39) Geneviève

MAZO, Le centre d'observation et la loi du 27 juillet 1942 relative à l'enfance délinquante. Thèse pour le doctorat en droit, éd. Van Etten, Paris, 1944, p 37.

(40) Voir la circulaire d'Armand Camboulives sur "La terminologie en matière pénitentiaire du 25 février 1940”, Union des sociétés de patronage, Recueil des bulletins 1941-1943 ; et la loi du 23 août 1940 qui rebaptisait les MES en IPES.

(41) JO, 13 août 1942.
Malgré des tentatives de réforme réglementaire au cours des vingt années précédant la guerre, rien n'avait fondamentalement changé au sein des établissements de l'Administration pénitentiaire. La guerre avait ensuite engendré une profonde désorganisation dans la prise en charge des mineurs. Quartiers spéciaux des prisons, colonies correctionnelles et maisons d'éducation surveillée avaient dû être fermés ou évacués. ${ }^{(38)}$ En 1940, la prison de Fresnes fut en partie réquisitionnée par l'armée allemande. Le nombre des mineurs incarcérés atteignit 325 pour une capacité de 240 cellules. ${ }^{(39)}$ Devant cette situation, un centre de détention préventive fut ouvert hâtivement, le 1er avril 1941, pour les garçons relevant du tribunal de la Seine, au 19 rue de Crimée à Paris. Quant aux établissements de redressement, après la fermeture définitive de la colonie d'Eysses le 15 août 1940, il n'en restait plus qu'un seul pour les filles et quatre pour les garçons. Cependant, dès le début de l'année 1940, des modifications importantes étaient engagées, symbolisées par des changements terminologiques. ${ }^{(40)}$ Alors que l'Éducation surveillée n'était encore qu'une simple sous-division de l'Administration pénitentiaire, Jean Bancal préconisait de construire une «cloison étanche " ou de procéder à " une opération chirurgicale " entre les deux administrations, les établissements et le personnel. Par ailleurs, considérant les activités des tribunaux pour enfants, Bancal considérait qu'un "juge bien informé» serait un remède efficace à l'usage aléatoire qui était fait du discernement.

Les deux courants n'étaient pas en opposition sur tous les points et se rejoignaient, notamment sur les perspectives de confier les jugements à un seul magistrat spécialisé et de permettre aux femmes d'exercer dans la magistrature. Ces idées, qui avaient été introduites de part et d'autre dans les différents projets antérieurs, n’ont pas été retenues par la loi. Sans doute ces questions n'étaient-elles pas "mûres", ou du moins apparaissaient-elles secondaires par rapport aux difficultés du moment qui poussaient à établir d'autres priorités.

\section{Les orientations politiques de Vichy face à la désorganisation sociale}

Typique du cadre idéologique de la Révolution nationale, le Rapport au Maréchal de France ${ }^{(41)}$ qui précédait le texte législatif affirmait la volonté de 
faire de la jeunesse française "une jeunesse forte, saine de corps et d'esprit, préparée aux tâches qui élèveront les âmes". Au lendemain du traumatisme de la défaite et de la panique des élites, c'est la volonté de ramener la paix et l'ordre à l'intérieur du pays qui provoqua la décision de l'armistice du 25 juin 1940. Un régime de type charismatique autour de la personnalité de Philippe Pétain se mit en place, alors que s'installait une collaboration d'État entre la France et l'Allemagne jusqu'en novembre $1942^{(42)}$ (avant la période collaborationniste). Les préoccupations qui soustendaient la législation de juillet 1942 étaient, certes, liées à l'augmentation de la criminalité juvénile qui sévissait en particulier dans les centres urbains, mais elle était amplifiée par la désorganisation due à la guerre.

Le parc des institutions susceptibles de prendre en charge les mineurs délinquants souffrait d'une grande pénurie. D’une part, en région parisienne, plusieurs établissements privés avaient été fermés ; les services sociaux étaient occupés à l'évacuation des populations et au transfert des enfants placés vers la zone libre. D'autre part, l'état des prisons était devenu catastrophique. Suite aux bombardements, des établissements pénitentiaires avaient été évacués, alors que le nombre de prisonniers avait triplé entre 1938 et $1942,{ }^{(4)}$ du fait des internements politiques. Les quartiers réservés aux mineurs avaient dû être libérés pour les adultes. Le phénomène de débordement des effectifs se doublait de l'exode des détenus et du personnel, afin de soustraire ces populations aux bombardements en zone de combat et d'éviter d'éventuelles libérations par les Allemands. Enfin, des écoles avaient également fermé leurs portes, faute de chauffage. Des enfants, victimes des dislocations familiales, erraient dans les rues, ou maraudaient, parfois avec l'acquiescement tacite des parents, sinon leur complicité, car l'urgence portait avant tout sur le ravitaillement, avec toutes les tentations qui en résultaient. ${ }^{(4)}$

Le Dr Petchot-Baquet, du Commissariat général à la famille, déclarait, en janvier 1942, que le nombre d'enfants criminels était passé de 15.000 en 1937 à 45.000 en 1940 et que, depuis, il ne cessait de croître. ${ }^{(4)}$ Il constatait un relâchement général des mœurs et s'alarmait sur le sort des fruits d'unions passagères ou irrégulières, qui seraient contaminés par l'alcoolisme et la débauche. Cette enfance-là, moralement abandonnée,
(42) D’après Jean-

Pierre AZEMA, "Vichy, repères historiques", Les cabiers de l'animation, $\mathrm{n}^{\circ} 49-50$, Institut national d'éducation populaire, ministère de la Jeunesse et des Sports, avril 1985.

(43) Pierre PEDRON, op. cit., pp. 63-64.

(44) D'après le rapport d'activités du Service social de l'enfance de Paris, années 1940-1942, archives de l'association Olga Spitzer.

(45) Henri GAILLAC, op. cit., p. 361, dénombre 11879 enfants jugés en 1937 et 34781 en 1942 ; Hélène CAMPINCHI, op. cit., p. 163, compte 1650 mineurs jugés en 1935 et 3452 en 1942 au TEA de la Seine. 
(46) AN, AG 2520.

(47) AN, AG 2 605,

Rapport du Dr Massé, 1939, adressé à Sautriau pour étude en avril 1941. deviendrait à coup sûr une jeunesse délinquante et révoltée.

Par ailleurs, le contre-amiral Bard, préfet de police, ${ }^{(46)}$ mesurait régulièrement la température de Paris. La peur des dirigeants portait sur les effets des privations imposées à une population où se multipliaient les suicides et les abandons d'enfants. C'était, selon lui, un terrain favorable à l'agitation communiste ou gaulliste. Le préfet reprochait à la magistrature de ne pas être assez répressive. Il soupçonnait les milieux communistes d'utiliser les éléments féminins non-communistes pour déclencher des campagnes d'agitation en faveur des prisonniers et de l'enfance.

Une autre question tourmentait les acteurs de la Révolution nationale : le problème récurrent de la dénatalité. Il était d'autant plus préoccupant que les années 40-41 enregistraient une baisse importante des naissances et que le dynamisme démographique de la France était très inférieur à celui des voisins italiens et allemands. Constatant que les dirigeants de ces deux pays ne se donnaient même pas la peine de dissimuler tous les espoirs malveillants qu'ils fondaient sur la dénatalité française, les membres du gouvernement de Vichy adoptèrent par mimétisme défensif cette conception de Mussolini : "Les peuples féconds sont des peuples forts et inversement les peuples stériles sont faibles. Lorsque ces derniers seront réduits à un amas de misérables vieillards, ils plieront à bout de souffle sous la poussée d'un jeune maître ".(7) Parallèlement au limogeage des instituteurs et des inspecteurs d'académie suspectés d'agitation politique et à l'épuration d'une magistrature jugée trop laxiste, les dirigeants de Vichy bâtissaient un ordre social centré sur la cellule familiale comme base indispensable au bien-être de la Nation et de l'État. Pour ce faire, ils encourageaient l'expansion du corps médical et son organisation corporatiste. En complément, ils déployaient des politiques d'encadrement de la jeunesse et favorisaient le développement des chantiers de jeunesse et des Compagnons de France, tout en y exerçant une surveillance active.

Pour régler la question de l'enfance délinquante dans ce désordre ambiant, deux projets furent élaborés en parallèle et sans concertation préalable officielle, entre 1940 et 1941. L'un fut échafaudé par la direction de la Famille sur l'initiative du médecin-commandant Sautriau, de Jacques Chevalier, de maître Garcin et du docteur Petchot-Baquet. Ce 
projet visait l'enfance coupable sous l'angle de l'enfance moralement abandonnée, dans la perspective d'une refonte du code de la famille. ${ }^{(48)}$ L'autre avait été concocté par Jean Bancal, inspecteur général adjoint des services de l'Administration pénitentiaire, et par Fernand Contencin, directeur de l'Administration pénitentiaire et des services de l'Éducation surveillée. ${ }^{(49)} \mathrm{Ce}$ dernier projet avait été réalisé sur la demande du ministre de la Justice, Joseph Barthélemy, qui entreprenait une réforme d'ensemble de l'Administration pénitentiaire.

\section{Deux projets en concurrence}

- Un code de l'enfance intégré au code de la famille, avec le Tribunal pour enfants et adolescents (TEA) comme auxiliaire ${ }^{(50)}$

En septembre 1940, ${ }^{(51)}$ le bureau "Enfance" de la direction de la Famille avait officiellement la charge de la petite enfance, de l'enfance malheureuse, des mineurs prostitués, des vagabonds, de la correction paternelle et de la surveillance des enfants placés. Il réclamait, entre autre, les mineurs délinquants, avec cette précision : "Ils dépendent actuellement $d u$ ministère de la Justice ; ily aurait le plus grand intérêt à confier à la direction de la Famille, déjà chargée des mineurs vagabonds, le relèvement social de ces enfants", car "l'enfance est UNE et [...] l'enfance délinquante n'est pas par nature distincte de l'enfance prédélinquante ». Cette orientation, préconisée par Garcin et de Lagrange, s'inscrivait dans "une refonte méthodique $d u$ code de la famille ", estimée urgente en raison des conséquences regrettables que la loi du 29 juillet 1939 avait produit sur la distribution des allocations familiales. ${ }^{(52)}$ Le secrétariat d'État à la Famille et à la Santé avait entrepris la réalisation d'un système complet de législation sur la famille, qui prévoyait une division en trois unités : civile, économique, sociale. Toute la difficulté, d'après Jacques Chevalier, était de "refondre sur le plan familial la législation individualiste issue de la Rérolution et du code civil ». ${ }^{(53}$ Déclarant que le secrétariat d'État à la Famille et à la Santé était le seul parmi les services gouvernementaux à disposer du personnel nécessaire - directeurs de la santé et de l'assistance, délégués régionaux à la famille, médecins, assistantes sociales, etc. -, Jacques Chevalier considérait qu'il fallait doter en crédits les services sociaux et les œuvres pri-
(48) AN, AG 2 605, textes du code, projets de refonte, étude des difficultés d'application, suggestions, notes.

Malgré plusieurs plans, ce projet n'aboutit pas à un nouveau code, mais à des modifications du code de 1939.

(49) Directeur de l'Administration pénitentiaire depuis le 30 août 1940 et successeur de Camboulives, nommé directeur des Affaires civiles et du Sceau.

(50) AN, AG 2 605, notes de Chevalier et exposé du Dr PetchotBacquet.

(51) Loi du 18 septembre 1940 relative à l'organisation et à l'action du secrétariat d'État à la Famille et à la Santé.

(52) Les familles nombreuses et nécessiteuses étaient défavorisées.

(53) AN, AG 2 605, Chevalier à Sautriau, 10 juin 1941. 
(54) Ibid.

(55) AN, AG 2 605, d'après le Dr PetchotBacquet, janvier 1942. Ce système est également décrit dans l'ouvrage de Michel CHAUVIÈRE, op. cit., p. 48 . vées, et notamment multiplier les assistantes sociales spécialisées près des tribunaux. Il défendait l'idée selon laquelle le pays et les œuvres devraient "perdre l'habitude de tout attendre de l'État ", mais, partant du constat que "l'initiative privée [avait] déjà pris une large part de l'assistance de la famille et de l'enfance ", il considérait que l'État se devait "d'empêcher les œuvres qui se trouvent en difficulté de disparaître, de donner à l'initiative privée un nouvel élan, de saisir le pays par une propagande active et soutenue de la question de vie ou de mort", qui se posait devant lui en ces termes : "natalité - Famille ". ${ }^{(54)}$

Famille et Justice devaient être deux administrations solidaires, selon le principe suivant : le TEA devenait l'auxiliaire de la famille. Sur cette instance centrale devaient aussi s'articuler les services sociaux, la police féminine, les médecins, les centres d'observation et de triage, et enfin les centres de rééducation. Le tout devait constituer un ensemble technique, ${ }^{(55)}$ dont l'unité serait assurée par des "Conseils de protection de l'enfance” (CPE), qui réaliseraient toute l'œuvre de prévention avant l'entrée en action du tribunal. Dès le début de l'instruction, l'enfant pourrait être placé dans un centre d'accueil et de triage. Les enfants déjà aux prises avec la Justice seraient envoyés dans des centres d'observation et de triage, où ils seraient longuement observés afin qu'un diagnostic soit établi, avant d'être dirigés vers le centre de traitement ou de rééducation approprié. Il s'agissait de centres médico-sociaux, (et non pas de centres de Justice) destinés à former "la pièce maîtresse du système de prophylaxie morale applicable à l'enfance en danger moral». Les Conseils de protection de l'enfance seraient institués à tous les échelons administratifs du pays. Le TEA serait composé d'un juge unique, qui pourrait être assisté d'un médecin, d'une femme qualifiée et d'un membre de l'enseignement ; le juge serait membre de droit des CPE.

Néanmoins, le rapport de Petchot-Baquet concevait que des mesures de répression puissent être infligées aux plus pervertis, sauf en cas de déficience mentale ou de défaillance parentale. Dans ce cas, l'internat de redressement pouvait éventuellement appartenir à l'administration de la Justice, mais le mineur n'en échapperait pas pour autant au contrôle du secrétariat d'État à la Famille et à la Santé. En modèle du système 
de rééducation, il était proposé : 1) au stade de la prévention de la délinquance, l'ESSOR occitan de l'abbé Plaquevent ; 2) immédiatement après le délit, les établissements de Mme de la Morlais, qui avait adjoint aux services sociaux de la cour d'appel de Caen et de Rennes les fermes rééducatives du Hinglé ; 3) après la punition, l'œuvre du père Aune, "L'étape", située en Provence, qui réhabilitait par le travail et l'acquisition d'un métier les jeunes libérés.

Pour appliquer cette politique, il fallait avoir les coudées franches afin de lutter contre les prérogatives des ministères qui, revendiquant " des attributions sur tels ou tels aspects du problème, [...] agissent isolément, sans coordination, sans direction commune ». La réorganisation de l'Éducation nationale et l'encadrement des chantiers de jeunesse venaient compléter ce vaste programme, pour lequel Jacques Chevalier avait réclamé "qu'aucun texte de loi ou décret ne soit publié sans avoir reçu le visa du secrétaire d'État à la Famille".

Les résistances allaient provenir de la Justice qui, de son côté, travaillait à sa propre réforme.

\section{- Un projet de loi portant code de l'enfance délinquante, ${ }^{(5)}$ sur le modèle de l'Éducation surveillée}

Du côté de l'Administration pénitentiaire, il s'agissait de lutter contre cette tendance qui amenait la confusion de toutes les catégories de mineurs et de conserver "l'enfance délinquante" en s'inscrivant dans la continuité de l'élan réformiste de 1936. Joseph Barthélemy avait entrepris une réforme d'ensemble de l'Administration pénitentiaire, dont la réalisation était devenue aléatoire en raison des troubles conjoncturels. Mais l'Éducation surveillée, moins directement affectée, pouvait recevoir quelques retouches. Aussi accorda-t-il une priorité à la question de l'enfance délinquante, pour laquelle il préconisait «d'abandonner résolument la conception répressive traditionnelle $»{ }^{(57)}$ Le ministre avait confié les travaux préparatoires à Bancal, "à cause de sa thèse ", ${ }^{(58)}$ et à Contencin, "en raison de son zèle $"{ }^{(5)}$ Des correspondances écrites avaient eu lieu entre la direction de l'Administration pénitentiaire à Vichy et le directeur des services à Paris, M. Papot. Par ailleurs, le directeur de la
(56) AN, BB 301711.

(57) Pierre PEDRON, op. cit., p. 87.

(58) Jean BANCAL, Essai sur le redressement de l'enfance coupable, Paris, Sirey, 1941, 82 p.

(59) AN, BB 30 1711, Discours de Joseph Barthélemy à la réunion du Conseil supérieur de l'Administration pénitentiaire, le 26 septembre 1941. 
(60) Mme de

La Morlais était inspirée par les idées d'Alexis

Carrel. Elle affiliait son service social près du tribunal au Service social de l'enfance de Paris. Si le SSE est resté silencieux durant cette période, c'est parce qu'il était affecté par une pénurie de personnel, de moyens financiers, et atteint par les politiques antisémites de Vichy.
Pénitentiaire avait été informé, par Jean Renaudin, du projet du secrétariat général à la Famille et à la Santé, des expériences modèles du secteur privé et des velléités d'expansion des services de Mme de la Morlais. Celle-ci avait écrit directement au Maréchal Pétain, pour proposer l'application de son système à toute la France. ${ }^{\left({ }^{(0)}\right)}$ Le directeur de la Pénitentiaire considérait qu'il fallait remédier sans retard à ce véritable "état d'anarchie ». Selon lui, la question de l'enfance délinquante se trouvait «fâcheusement compliquée par beaucoup de confusion dans les idées et dans les textes [...] et par le zèle parfois intempestif de bonnes volontés, le plus souvent idéalistes et généreuses mais trop fréquemment aussi incompétentes, susceptibles ou même intéressées, et enfin par un excès de candeur et de sensiblerie peu compatible avec une saine appréciation des réalités". Il adressait une sévère critique aux établissements de type "Bon Pasteur", parce qu'ils favorisaient la contamination en mélangeant toutes les catégories. Il estimait que toutes ces œuvres privées étaient désireuses de tirer les meilleurs profits possibles : "il s'ensuivrait fatalement des rivalités et des luttes d'influence auprès des juridictions pourvoyeuses pour obtenir de celles-ci la faveur d'affectations selon leurs désirs". Il ajoutait que "l'ingérence sans cesse accrue de leurs représentants dans l'auvre de justice, ainsi que leur tendance à pousser à une extension croissante du champ de recrutement des mineurs par autorité de justice [...] contribuait à l'accentuation progressive de la confusion de la situation des mineurs de justice et des autres ».

Devant les prérogatives de la Famille, garder les enfants traduits en Justice, alors que les crédits d'équipement étaient quasi inexistants, était un pari difficile. Dans cette configuration, la solution fut de recourir au système du modèle et de la doctrine. Créer peu de centres, mais bien les équiper, déployer des méthodes efficaces, réorganiser les IPES et les colonies correctives... bref, montrer l'exemple, voilà ce qui permettrait à l'Éducation surveillée de conserver ses attributions dans un contexte aussi peu favorable. Deuxième condition : les œuvres privées devaient se soumettre à une discipline, se spécialiser en catégories de mineurs ou en types d'activité (artisanat, agriculture...) et s'astreindre à des contrôles.

Le texte législatif élaboré par Bancal et Contencin au début de l'année 1941 était assez proche de la version finale, mais il n'avait intégré ni 
les prétentions, ni les préoccupations des protagonistes du système famille/TEA. Par rapport à la forme définitive de la législation, l'esprit du premier projet était plus répressif et son écriture laissait moins de flou juridique. Le texte avait été annoté de commentaires manuscrits qui comportaient notamment le dénombrement suivant : " 20.000 infractions d'enfants dont 5 à 6.000 sérieux, 2.000 en maisons d'Éducation surveillée ». Les rédacteurs du code prévoyaient d'abaisser la majorité pénale à 16 ans, considérant qu' "aucune auvre éducative n'est possible à partir de certains âges ". Si l'idée de supprimer les sanctions répressives était avancée, des restrictions subsistaient pour les mineurs de 16 ans convaincus de crime et d'avoir agi "avec discernement". Ceux qui seraient passibles de la peine de mort, des travaux forcés à perpétuité et de la déportation encourraient une condamnation à la peine de 10 à 20 ans d'emprisonnement dans un quartier spécial d'une colonie correctionnelle. ${ }^{\left({ }^{(1)}\right)}$ En outre, l'intention d'instituer la chambre du conseil en première phase de la procédure avait été annotée "très bien ". La limitation des voies de recours était commentée ainsi : "on fera examiner la culpabilité par la chambre du conseil et les mesures seront toujours provisoires ; c'est analogue à une garde d'enfant, c'est une juridiction spéciale. On peut toujours revenir par l'incident à la liberté surveillée. Ce tribunal ne prononce pas de peine, simplement des mesures sur lesquelles il est possible de revenir ". Car, en fait, l'essentiel de la réforme reposait sur la conviction qu'une autorité judiciaire spécialisée, sollicitée à bon escient, équipée de centres d'observation et connectée à un dispositif public et privé, allait être le remède miracle. Il fallait faire confiance à cette juridiction, puisqu'il lui était possible de revenir sur les jugements. Les procédures de défense devenaient donc superflues.

Si l'Administration pénitentiaire avait été mise au courant des projets du commissariat général à la Famille au cours du premier trimestre 1941, ce dernier, en revanche, semblait ignorer les perspectives de réforme de la Justice. De ce fait, la question de l'enfance délinquante prit l'allure d'une querelle de monopole. Les négociations pour le partage des pouvoirs se déroulèrent au sein du Conseil supérieur de l'Administration pénitentiaire, en septembre 1941, à Vichy.
(61) Ce point

n'apparaît plus dans le texte final. 
(62) AN, AG 2605

CM 19 D.

(63) Décret du 4 septembre 1940, Union des sociétés de patronage, Recueil des Bulletins 19411943, p. 188.

(64) Il s'agit de Pierre Ceccaldi.

\section{La résolution du Conseil supérieur de l'Administration pénitentiaire}

Considéré comme un recul, un retour à des méthodes répressives, coûteuses, inopérantes et retardataires par rapport aux législations étrangères (Scandinavie), le code de l'enfance délinquante fut vivement critiqué par les défenseurs de "l'enfance moralement abandonnée". gés par le fait que les grands experts, tels que Henger, Magnol, Donnedieu de Vabres, Lespinasse, Riser n'avaient pas été consultés, et considérant que la différence entre enfance délinquante et prédélinquante ne présentait "qu'un intérêt spécifiquement judiciaire », les protagonistes de la Famille préconisaient un ajustement avec leur propre projet de loi. La chose fut faite le 26 septembre 1941 dans une salle de l'hôtel Carlton à Vichy, mais dans le camp de la Justice. En effet, sous la présidence de Joseph Barthélemy, le Conseil supérieur de l'Administration pénitentiaire donna à la législation sa tournure définitive au prix de quelques altercations.

La fonction qui fut dévolue au Conseil supérieur de l'Administration pénitentiaire en ce mois de septembre 1941 fut celle d'une instance parlementaire délestée de toute considération politique. Restructuré par le décret du 4 septembre 1940, cet organisme rompait avec une organisation plus ancienne, "le Conseil supérieur de prophylaxie criminelle", et venait remplacer les trois organismes consultatifs qui fonctionnaient depuis 1935 auprès du ministère de la Justice. Il avait été conçu pour être orienté avant tout vers l'action. Pour ce faire, il avait été considérablement allégé. Des 64 membres précédemment concernés, l'effectif avait été réduit à 24 membres de droit, un président et un vice-président. ${ }^{\left({ }^{(3)}\right.}$ Bien que l'appareil judiciaire y fut fortement représenté, la composition du groupe permettait d'établir un lien indispensable entre les divers départements ministériels. Ce conseil réunissait des représentants de la Justice : MM. Pernot, Dayras, Camboulives ; de la Famille : M. Denis ; de la Jeunesse : M. Le Guen ; de l'Enseignement technique et primaire : MM. Froment et Joly ; des membres de l'Administration pénitentiaire et de l'Éducation surveillée : MM. Contancin, Dequiat, Bancal, Pinatel, Matthieu, Ceccaldi ${ }^{(6)}$; des magistrats du tribunal de la Seine : MM. Baffos, Cenac, Perrier, Bashet (les trois derniers étaient excusés) ; 
des représentantes des services sociaux attachés aux tribunaux : Mmes Guichard (Sauvegarde de l'adolescence de Paris), et Spitzer (SSE Paris, excusée) ; des experts en droit : MM. Donnedieu de Vabres, Legal, de Casabianca (excusé) ; des experts en psychiatrie juvénile : $\mathrm{M}$. Heuyer et Mme Badonnel (section de préservation de Fresnes, excusée).

Le ton fut donné par Joseph Barthélemy : il s'agissait de spécialiser la prise en charge de l'enfance délinquante dans un souci de clarté et de réalisme. L'essentiel de la discussion porta sur l'abaissement de la majorité pénale et sur les velléités du Commissariat général à la famille.

Sur le premier point, c'est Heuyer, en expert astucieux, qui fit pencher la balance en faveur du maintien de la majorité pénale à 18 ans : " 16 ans, c'est trop tôt, la puberté n'est pas finie [...] l'orientation professionnelle est encore possible [...] si vous passez la majorité pénale à 16 ans, c'est, d'après mon enquête, ${ }^{(6)} 26$ à $37 \%$ des délinquants qui vont vous échapper!" Face à cet argument, les résistances tombèrent partiellement. Il fut décidé de maintenir des sanctions spécifiques pour les 16-18 ans, mais la question de l'application des peines fut éludée. Dans un courrier postérieur à cette réunion, Ceccaldi avait donné quelques précisions quant aux lacunes juridiques concernant l'application des peines (qui auraient pu être : mort, travaux forcés à perpétuité, déportation, dégradation civique). En réponse à la virulente critique de Joseph Magnol qui, en octobre 1942, avait demandé une révision de la législation, Ceccaldi indiquait que l'omission des peines avait été volontaire : "Ces peines criminelles sont en principe applicables aux mineurs qui les ont encourues sans aucune atténuation légale. Le législateur aurait voulu laisser peser une menace sur les enfants les plus pervers. En fait, le tribunal pour enfant n'appliquerait jamais cependant la peine criminelle ".

Le deuxième point fut abordé sans détour par le ministre de la Justice : "La Famille réclame-t-elle le jugement de l'enfant délinquant? " Les échanges furent tendus, mais la résolution émergea sous la pression de Richard, représentant des Finances. Déclarant que trois ministères étaient concernés par l'enfance délinquante (Jeunesse, Famille, Justice), il souhaitait que ne s'organisent pas trois systèmes. Comme une conciliation approfondie lui semblait impossible à obtenir, Richard proposa plusieurs fois que l'on ajourne les questions d'organisation, mais l'idée d'un ajourne-
(65) Georges

HEUYER, "Enquête sur la délinquance juvénile, étude de 400 dossiers", in Pour l'enfance coupable, 1942. 
(66) Loi $\mathrm{n}^{\circ} 815 \mathrm{du}$

26 août 1942. JO,

29 août 1942, p. 2954. ment faisait craindre des dépenses supplémentaires en raison de l'élévation du prix des constructions. Les différents protagonistes s'orientèrent alors vers une solution médiane et économique : la réciprocité. "Plutôt que de multiplier les centres, coordonnons ! ", suggéra Barthélemy. Il fut donc décidé de modifier l'article 27 : les centres d'observation contrôlés par la Famille et la Santé pourraient être utilisés par la Justice et inversement. En contrepartie, les Finances voteraient les crédits pour l'ouverture de trois centres d'observation au ministère de la Justice. Par économie également, il fut décidé de ne pas systématiser les enquêtes sociales et les examens médicaux. Il aurait fallu pourvoir tous les territoires juridictionnels en personnel et en locaux. Or le conseil considéra que la province n'aurait jamais les moyens de s'équiper comme à Paris.

Le 21 mars 1942, Joseph Barthélemy adressait aux procureurs généraux une circulaire stipulant qu'ils devaient inviter leurs substituts « $\grave{a}$ rechercher, au siège du tribunal ou dans les environs immédiats, un établissement public ou privé, ou encore une personne charitable, susceptible de prendre l'enfant en attendant que l'autorité judiciaire statue sur son cas". Il signalait "les possibilités que peuvent offrir les foyers dépositaires de l'Assistance publique, ainsi que les hôpitaux".

Le 31 mars 1942, Yves Bouthilier, ministre des Finances, informa les services de l'Administration pénitentiaire de Paris que la somme de 2.942.650 F serait allouée pour le fonctionnement des cinq TEA et des six centres d'observation en 1943. Cette somme incluait une reconduction du budget de 1942, car seuls trois centres provisoires avaient fonctionné.

Un mois après la promulgation de la loi, le chef du gouvernement décrétait que la coordination entre les différentes administrations concernant l'enfance déficiente et en danger moral serait assurée sous sa responsabilité directe, par délégation des pouvoirs aux secrétaires d'État. ${ }^{(66)}$

Dorénavant, tant que le traitement de l'enfance délinquante conserverait un versant pénal, comme le stipulait la loi du 27 juillet 1942, l’Éducation surveillée avait toutes les chances de conserver ses attributions sous la tutelle de la Justice, et ce, malgré les velléités du ministère de la Santé après la Libération. 


\section{Conclusion}

Ce serait une erreur d'isoler la loi du 27 juillet 1942, comme si elle était un avatar du régime de Vichy. Cette loi s'inscrit dans une continuité par rapport aux évolutions antérieures, mais les conjonctures liées à la défaite ont précipité les décisions politiques. La faiblesse des budgets de l'État, l'accroissement de la criminalité, la désorganisation sociale et les concurrences institutionnelles ont poussé le législateur à des compromis difficiles et à des arrangements pragmatiques à moindre coût, à tel point que l'application législative fut presque nulle. Cette loi eut toutefois un effet durable sur le traitement de l'enfance délinquante, car elle en affirmait le versant pénal tout en introduisant une nouvelle conception de la minorité pénale. Cette orientation ne fut jamais remise en question, même par Hélène Campinchi, pourtant sensible à la cause des enfants “en danger moral”, lorsqu'elle fut chargée, après la Libération, de préparer l'ordonnance du 2 février 1945. En ce sens, et sur plusieurs autres points (la suppression du discernement, la spécialisation des juges, l'observation du mineur, le contrôle des établissements privés), la loi de 1942 préfigurait l'ordonnance de 1945 . $^{(67)}$ Du côté de la protection civile, le courant de dépénalisation de l'enfance coupable se poursuivit pendant les années de guerre par des programmes de rééducation et de prévention étayés sur des conceptions médicales et familialistes. Les docteurs Heuyer et Lafon, le ministre Grasset et le juge Chazal entérinèrent cette politique, déjà visible en 1942, lorsqu'ils instaurèrent le Conseil technique de l'enfance déficiente et en danger moral, en juillet 1943. Ce dispositif conforta la suprématie du domaine de la Santé sur l'enfance inadaptée. Certaines questions restèrent en suspens jusqu'à la Libération : l'intervention d'un juge unique, l'entrée des femmes dans la magistrature, ${ }^{(68)}$ l'émancipation et la restructuration de l'Éducation surveillée ${ }^{(69)}$; ces projets de réforme, conçus avant la guerre, trouvèrent des résolutions partielles sous Vichy : une collégialité de juges spécialisés, des assesseurs éventuellement féminins, l'entreprise de différencier l'Éducation surveillée de l'Administration pénitentiaire. Lorsque cet élan réformiste aboutit grâce aux nouvelles conjonctures de 1945, les rivalités structurelles entre les institutions de la Santé-Population et de la
(67) Voir l'analyse de Philippe ROBERT,

Traité de droit des mineurs, IME, Besançon, 1969, pp. 95-96.

(68) Ces deux points, évoqués le 21 septembre 1941, furent rejetés par la majorité des membres du conseil. La présence des femmes dans la juridiction était toutefois envisagée par le biais des "assesseurs" en cas de crime.

(69) Michel CHAUVIÈRE, "L'émergence de l'Éducation surveillée en France...vers 1945", in Michel CHAU-

VIÈRE, Pierre LENOËL et Éric PIERRE, Protéger l'enfant, Rennes, PUR, 1996, pp. 149-164. 
(70) Philippe

ROBERT, op. cit., p. 95.
Justice n'en cessèrent pas pour autant. En effet, dans le contexte de la restauration d'après-guerre, il fut envisagé que l'Éducation surveillée passe sous la tutelle du ministère de la Santé. C'est dire combien le traitement de "l'enfance à problèmes" demeure une question récurrente, dépendante des enjeux nationaux, sans cesse soumise aux rapports de forces politiques et institutionnels. Comme chaque nouvelle loi s'élabore en fonction des contextes socio-économiques; elle se justifie en se proclamant plus simple, plus souple, plus progressiste que celle qu'elle remplace. Si la loi de 1942 voulait abolir le système de 1912 estimé "trop compliqué, trop lent, trop subtil", la nouvelle législation de 1945 dénonçait le caractère "réactionnaire, chimérique et compliqué de l'acte $d u$ gouvernement de Vichy ", pour annoncer qu'elle serait "libérale, pratique et simple ${ }^{\left({ }^{70)}\right.}$ Les dispositions de la législation de 1958, qui fondent le système dualiste actuel, s'établirent également selon ce mécanisme évolutionniste, caractéristique du droit positif. 